\title{
Dolphin sonar-modelling a new receiver concept
}

\author{
Peter Dobbins \\ Systems Engineering \& Assessment Ltd., SEA House, Bristol Business Park, Coldharbour Lane, \\ Bristol BS16 1EJ, UK \\ E-mail: peter.dobbins@sea.co.uk
}

Received 15 December 2006

Accepted for publication 14 February 2007

Published 14 March 2007

Online at stacks.iop.org/BB/2/19

\begin{abstract}
Observations suggest that dolphin sonars function well in the very shallow, reverberant, near-shore region of the ocean, and significantly out-perform man-made systems under such conditions. The echolocation characteristics of many small cetaceans have been measured directly and the high performance of biosonar systems is not in question, but explanations for their resolution, target detection, localization and tracking abilities are inadequate and deserve further investigation. The dolphin's lower jaw has been identified as part of an echo-receptor, and several hypotheses have been proposed to explain this. In one of these, the regularity of dolphin teeth was considered as a sonar array. This paper explores the physics of such systems with models based on established radar and sonar principles, and using data from various dolphin species. The insights gained from this modelling then lead to speculative proposals for new sonar receiver concepts that may have advantages over more conventional designs in shallow water operation.
\end{abstract}

(Some figures in this article are in colour only in the electronic version)

\section{Introduction}

There is a need for small, lightweight, high-resolution sonars capable of effective operation in the shallow, nearshore, littoral region of the ocean, to be carried by divers or underwater vehicles in naval mine clearance and other applications (Commander et al 2002, Hallett and Chapman 2003). In many current imaging systems, the high frequency used to achieve fine resolution limits the maximum range to tens of metres due to absorption but, even with focusing, nearfield effects dominate at shorter ranges (Belcher et al 2002). On top of this, surf zone and littoral operation is particularly difficult for any form of sonar because of reverberation, turbulence, suspended sediments and bubbles, and many other difficulties (Richards and Leighton 2003).

These environmental characteristics, however, had important consequences for the evolution of dolphins and other cetaceans. The highly cluttered, visually limiting but acoustically rich environment created selective pressures that favoured sound-based sensory systems capable of homing on edible prey, navigation, avoidance of predators and location and identification of other dolphins (Au 2004, Johnson 1997).
It is now understood that dolphins and other animals using acoustic echolocation can significantly out-perform man-made systems, as demonstrated, for example, by Sigurdson (1997a).

After 35 million years of trial and error, dolphins have evolved an echolocation system as the optimal sensor for detecting, classifying and localizing targets the size of a sardine (sardines (Sardina pilchardus) are typically 9 to $18 \mathrm{~cm}$ long with target strengths in the range -53 to $-48 \mathrm{~dB}$ (Mendes et al 2004)) over ranges from zero to $100 \mathrm{~m}$ or more in all sea states and all maritime environments from the open ocean through the surf zone to rivers and estuaries. These skills have certainly been established in open water (Sigurdson 1997a) and shallow water (Sigurdson 1997b). They have also been reported for some species of river dolphin (Ura et al 2006). Dolphins have been observed catching fish in the surf zone in extremely shallow water, but whether they are using echolocation is not known.

It is worthy of comment that, although dolphins appear to thrive in almost all aquatic environments, many of the river dolphins are amongst the most endangered species (Reeves et al 2003). In most cases this is due to pollution or habitat 

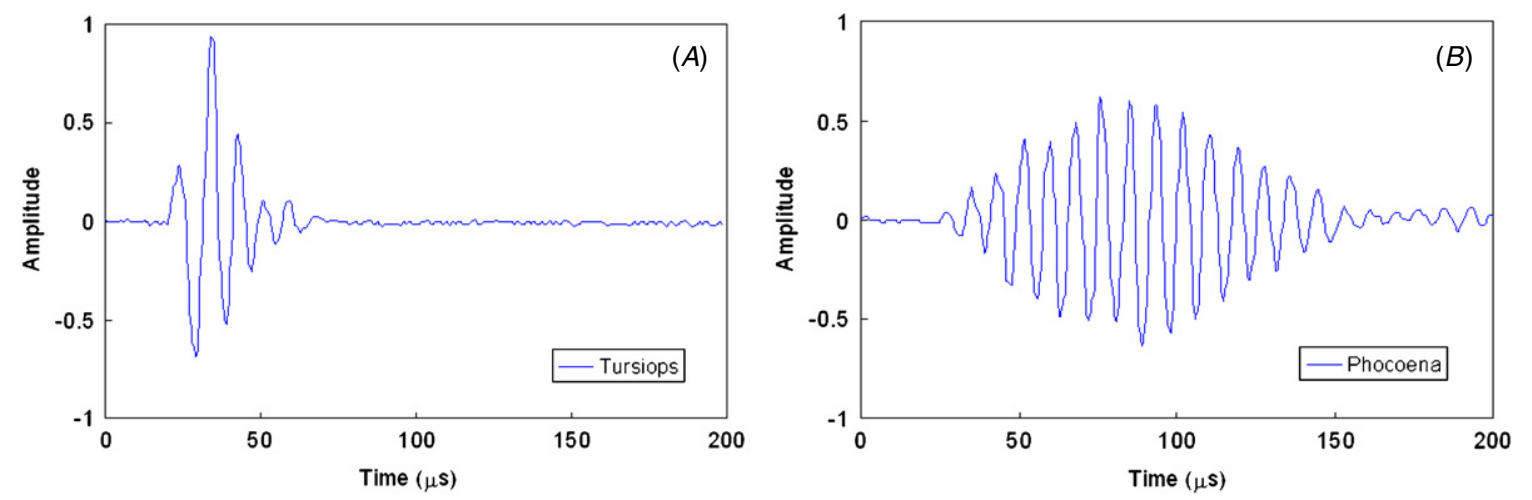

Figure 1. Examples of the echolocation signals of a whistling dolphin, T. truncatus $(A)$, and a non-whistling porpoise, $P$. phocoena (B).

deprivation, but it is difficult to understand why, in a river the size of the Ganges or the Yangtse, it is still not possible to find a niche. It may be that there are not sufficient prey species to sustain the dolphins, but it is also possible that the river dolphin's echolocation was only just able to detect and identify edible fish adequately in such difficult environments, but the newly added pressures of pollution and habitat deprivation have proved too much.

Dolphin echolocation, like any sonar, is a system consisting of many components, but essentially a transmitter, a receiver and a processor. This paper is mainly concerned with the receiving mechanism, along with the processing involved in beam forming and target localization. A wider discussion of dolphin echolocation and hearing will be found in the book by $\mathrm{Au}$ (1993) or a more recent collection of articles covering all aspects of echolocation (Thomas et al 2004).

There is evidence that the dolphin's lower jaw is a component in the echolocation receiver, and Goodson and Klinowska (1990) have proposed a model suggesting the equally spaced rows of teeth form receiving arrays. Various processing schemes for these 'tooth arrays' have been suggested (e.g. Potter and Taylor 2001) but, whatever the details, such arrays in an endfire mode, unlike broadside arrays, should maintain directivity in the near field (Berktay and Shooter 1973). In addition, combining the rows of teeth in a monopulse configuration would give fine angular resolution but with wide beams for rapid area searching (Dobbins 2001).

This model is based on the sea-going odontocetes (toothed whales) in general, and the Atlantic bottlenose dolphin, Tursiops truncatus (Goodson and Klinowska 1990, Dobbins 2001) in particular, but other cetaceans have evolved different solutions to the difficulties of sonar operation under shallow, reverberant and noisy conditions. One obvious difference is the range of transmission waveforms employed by various species. Another is the very different tooth and jaw structure of the river dolphins. The aim of this paper is to compare some of these different approaches and consider which, if any, might be applied in a bio-inspired sonar to solve the problems of shallow water operation.

\section{Dolphin signals}

The signals used for echolocation by whales and dolphins tend to fall into two broad categories, depending on whether the animal uses lower frequency whistle signals for communication. Those that typically emit whistles also emit brief broadband echolocation clicks having between four and eight cycles and durations of 40-70 $\mu \mathrm{s}$. Most dolphins fall into this class (Au 1993).

These signals do not change much in duration or shape, and the centre frequency is generally a function of the intensity of the transmitted signal, varying almost linearly with the level of the signal (Au et al 1995). Source levels up to $235 \mathrm{~dB}$ re $1 \mu \mathrm{Pa} @ 1 \mathrm{~m}$ have been measured for bottlenose dolphins (Tursiops truncatus) and such high intensity signals often have centre frequencies of $100 \mathrm{kHz}$ and higher, whereas very low intensity signals often have centre frequencies between 30 and $60 \mathrm{kHz}$.

Dolphins and porpoises that do not emit communication whistles use narrowband echolocation signals with at least 12 cycles and a duration generally greater than $100 \mu \mathrm{s}$ (Au 1993). Among odontocetes that emit signals in this category, the most common is the harbour porpoise (Phocoena phocoena) which employs source levels in the range 140$170 \mathrm{~dB}$ re $1 \mu \mathrm{Pa} @ 1 \mathrm{~m}$.

Examples of typical signals used by a $T$. truncatus (a whistling dolphin) and a $P$. phocoena (a non-whistling porpoise) are shown in figure 1 . These animals can detect small targets such as a $5 \mathrm{~cm}$ diameter sphere (target strength $-38 \mathrm{~dB}$ ) at ranges from a few tens of metres for the harbour porpoise (Kastelein et al 1999) to over a hundred metres for bottlenose and other large dolphins (Au 1993).

It should be noted that the echolocation signals of some species, including T. truncatus, can vary greatly for different tasks such as general searching or close examination of an object. Both the intensity and the spectrum can be changed and the signals seem to adapt to give the optimum available for the task in hand (Capus et al 2007).

The transmitting beam pattern for most odontocetes is sidelobe free and, in the case of the bottlenose dolphin, the broadband beamwidth is approximately $10^{\circ}$ in both horizontal and vertical planes (Au 2004).

\section{The tooth array concept}

The model proposed by Goodson and Klinowska (1990) is based on the observation that dolphins are homodonts, 

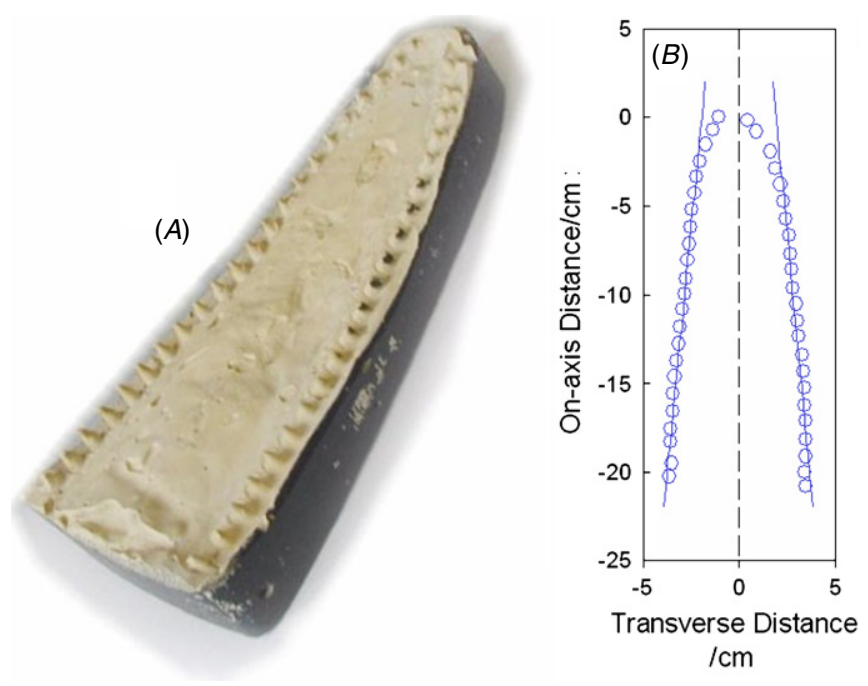

Figure 2. Plaster cast taken from the lower jaw of a bottlenose dolphin $(A)$ and positions of the teeth plotted in Cartesian coordinates $(B)$. The lines are linear fits to the teeth positions ignoring the most forward three teeth on either side.

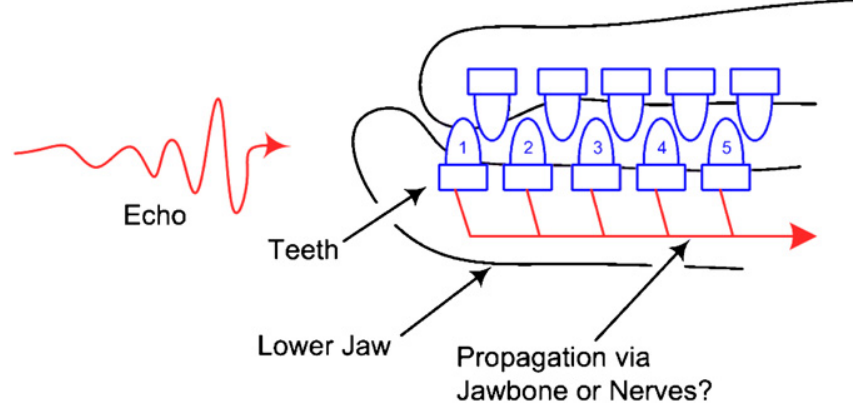

Figure 3. The endfire tooth array. Signals arriving from the forward direction are received by teeth and propagated either as nerve impulses to the central nervous system or as sound, via the jawbone, to the ear. Propagation delays cause signals from all teeth to arrive simultaneously (after Goodson and Klinowska (1990)).

that is their teeth are all of the same type, as opposed to heterodonts which possess incisors, canines, molars and so on. Furthermore, in each jaw (although some species have teeth in the lower jaw only) they are divided into two, essentially straight, equally spaced rows diverging at an angle in the order of $10-20^{\circ}$. These observations hold true for almost all the maritime odontocetes. An example can be seen in figure 2 . This shows a plaster cast taken from the lower jaw of a bottlenose dolphin on the left, and the positions of the teeth plotted in Cartesian coordinates on the right. A trend line is also plotted through the teeth on either side of the jaw (neglecting the most forward three teeth on either side), and this shows how close to a straight line the two rows of teeth are, except for the slight curvature near the front of the jaw.

In Goodson and Klinowska's (1990) model, it is postulated that the teeth act as resonant pressure transducers, which are combined as two equispaced line arrays with the tooth nerves introducing progressive propagation delays, creating a delay line beamformer as shown schematically in figure 3 . The nerves then transmit the auditory information directly to the central nervous system (CNS). Potter and Taylor (2001) examined the possibility of appropriate delays with realistic nerve conduction speeds and found that suitable delay line mechanisms had indeed been found in the auditory cortex, although it has yet to be shown that they could form part of a tooth-array beam former.

The biological mechanisms involved are not considered here, but the involvement of the tooth nerves is not essential. A more feasible concept is that the teeth act simply as passive resonators, coupling incoming acoustic waves into the jawbone, and then conduction through the jawbone provides appropriate delay paths, channelling the vibrations to the ear where they are perceived as sound. Many passive endfire arrays based on such principles exist, e.g. the TV crew's 'shotgun' microphone (Sank 1985) as well as the ubiquitous rooftop TV antenna.

The involvement of the lower jaw in dolphin echolocation reception is now widely accepted, and many experiments have been published that support this. Some have looked at auditory brainstem responses (ABR) to various stimuli in dolphins. Some have looked at sensitivity to external sound sources (Cook et al 2004), but many have attempted to map the response to acoustic stimuli applied directly to the head and jaws of dolphins (Brill et al 2000). The latter are relevant to this work, but exactly what the acoustic pathways are is not yet clear. None of these have applied stimuli directly to the teeth, and such an experiment may be informative. Potentially interesting work at Loughborough University using laser Doppler vibrometry (Dible et al 2006) is at an early stage, but shows promise and may eventually map acoustic pathways in dolphin teeth and jaws.

More than just the jaw bone, it has been realised for some time that the complex structures of fat and bone that make up the dolphin's jaw could form the necessary propagation channels. Recent research by Ryabov (2004) suggests that there are at least four independent channels within the mandibular canal on either side of the jaw, each filled with fat whose impedance is close to water. The cross section of these channels expands approximately exponentially towards the rear of the jaw, potentially providing an acoustic impedance match between the seawater and the ear.

If the propagation speed within the channels is close to that of water, they could provide the necessary delays for a tooth array, although how the sound is initially coupled into the channels from the teeth has yet to be explained.

Finally, as mentioned above, the acoustic characteristics of dolphin teeth are currently being investigated by Dible et al (2006). Using a laser Doppler vibrometry measurement technique, they have found strong resonant modes within the teeth of a T. truncatus in the band 115 to $135 \mathrm{kHz}$. This band overlaps the animal's known echolocation frequencies. Resonances have also been found in sperm whale (Physeter macrocephalus) teeth corresponding with their echolocation band (Nelson 2005). Additionally, it may be noted that the spacing between the teeth of the bottlenose dolphin is close to a wavelength at the highest frequency in their echolocation range.

Overall, it seems plausible that tooth array mechanisms exist in many of the odontocetes with the teeth acting as 


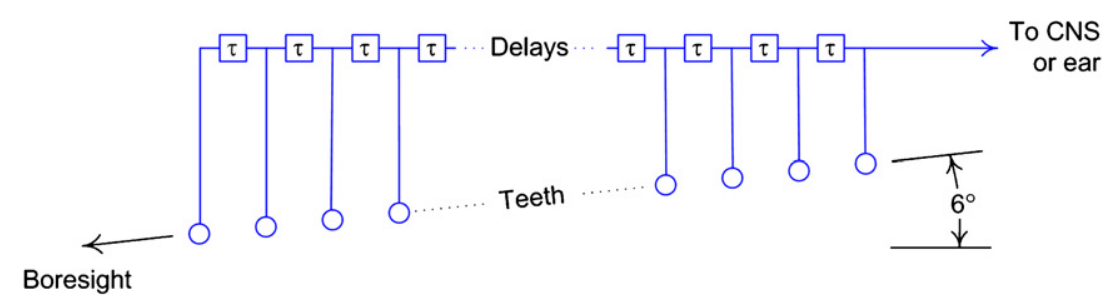

Figure 4. Sketch showing principle of a row of teeth acting as an endfire array beamformer.

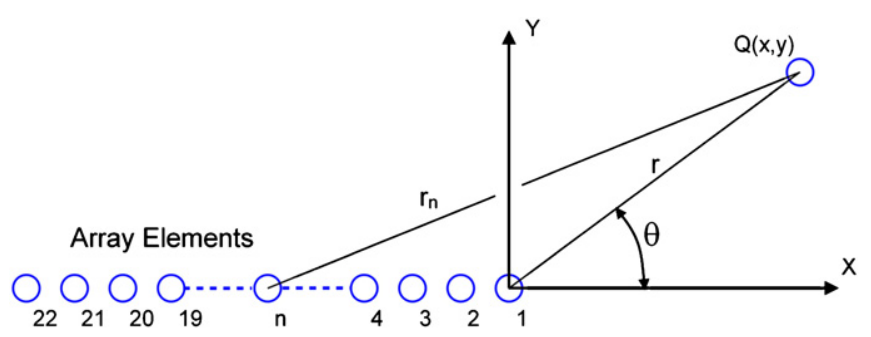

Figure 5. Sketch of array geometry and coordinate system.

resonant receivers arranged in geometries appropriate for beamforming, and with the required delays derived either neurologically in the nervous system or, more probably, acoustically in the fatty channels within the jaw bone. This hypothesis is only partially tested, but the work by Dible et al (2006) and others may lead to a complete test in the near future.

\subsection{Endfire arrays}

The endfire array is widely used in radio, radar and audio technologies (e.g. Sank 1985, Kraus and Marhefka 2002), but is not common in sonar systems. The principle of operation is shown schematically in figure 4. As suggested above in relation to figure 3 , the teeth act as pressure transducers. Signals representing sound pressure are transmitted either to the CNS along the mandibular nerves, or to the ear through the fat-filled channels in the jaw, with propagation delays ( $\tau$ in the diagram) such that an echo arriving from a direction along the row of teeth (boresight) results in all signals arriving at the CNS simultaneously and adding constructively. Signals from other directions will not arrive simultaneously, resulting in a reduced response. Thus, the array has directivity, with maximum sensitivity in the boresight direction.

The Tursiops lower jaw has two rows of about 22 teeth, spaced at approximately $1 \mathrm{~cm}$ and diverging at an angle of around $12^{\circ}$. Beam patterns for an individual line array were computed as follows.

For the purposes of computing the pattern, the array is considered to lie along the $x$-axis in Cartesian coordinates, as shown schematically in figure 5 . The front element, numbered 1 , lies at the origin. A source, $Q(x, y)$, is at range $r$ from the reference element 1 and a bearing $\theta$ relative to the $x$-axis. Clearly, $x=r \cos \theta$ and $y=\sin \theta$ and the distance from source to the $n$th element at $\left(x_{n}, y_{n}\right)$ is given by

$$
r_{n}=\sqrt{\left(x-x_{n}\right)^{2}+\left(y-y_{n}\right)^{2}} .
$$

In the beamformer arrangement outlined in figure 4, the delayed outputs from the receiving elements are added together to form a directional beam. If the delay is zero, a broadside beam results with maximum directivity normal to the line of the array. If, as in figure 4 , an incremental delay is applied to each element equivalent to the propagation delay over the distance between elements, then an endfire beam is obtained. In this arrangement, the delay at the $n$th element is

$$
\tau_{n}=x_{n} / c=(n-1) d / c,
$$

where $c$ is the sound speed and $d$ is the element separation distance. The beamformer output $B(r, \theta)$ is then given by

$$
B(r, \theta)=\sum_{n=1}^{N} \frac{1}{r_{n}} \exp \left[-\mathrm{i}\left\{k\left(r_{n}-r\right)-\omega \tau_{n}\right\}\right],
$$

where $\omega=2 \pi f, f$ is the frequency and $k=\omega / c$ is the wavenumber.

Equation (3) used the exact distance from the source to array element, so is valid for computing near-field beam patterns. It is, however, subject to a number of simplifying assumptions. Firstly, it assumes spherical spreading between the source and receiving element, but it is possible that structures in the vicinity of the receiving elements within the skull could distort arriving wavefronts and there may be mutual coupling between array elements. This could degrade angular localization performance because the apparent direction of arrival is in error because of distortion of the wavefront. The other significant limitation of this model is that it assumes continuous tonal signals. The dolphin's echolocation click is much shorter than the length of the array. For sources near the boresight, delays will ensure that the signals from all elements are aligned, but at wider, off-axis bearings this will not happen. This should not degrade angular localization, which is only important near the boresight, but is likely to degrade the sidelobe performance of the array, making it more susceptible to noise and reverberation. These factors deserve further investigation, but do not negate the basic concept.

Beam patterns for an unshaded endfire array with 22 elements and $1 \mathrm{~cm}$ spacing are plotted in figure 6 . This shows far-field patterns at frequencies of $50 \mathrm{kHz}$ and $120 \mathrm{kHz}$, the extremes of Tursiops' echolocation bandwidth, in figures 6(A) and $(B)$, respectively. These patterns are much the same as for a conventional broadside array, with a distinct main beam surrounded by several lower level sidelobes. The beamwidth is greater than that of a broadside array of the same length and reduces with increasing frequency from $40^{\circ}$ at $50 \mathrm{kHz}$ to $26^{\circ}$ at $120 \mathrm{kHz}$. 

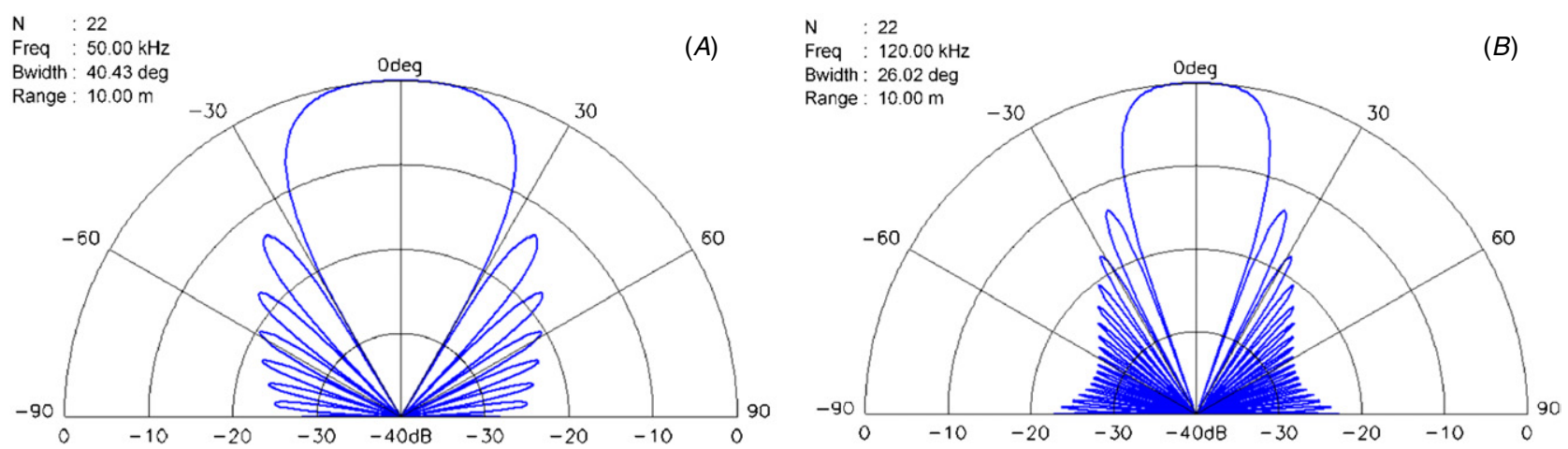

Figure 6. Endfire array far-field directivity patterns for Tursiops' tooth geometry plotted for $50 \mathrm{kHz}(A)$ and $120 \mathrm{kHz}(B)$.

However, as suggested by Berktay and Shooter (1973), it is at a short range that the endfire array differs most significantly from a broadside array. In figure 7 , endfire patterns at $120 \mathrm{kHz}$ for ranges of $3 \mathrm{~m}, 1 \mathrm{~m}, 0.3 \mathrm{~m}$ and $0.1 \mathrm{~m}$ are shown in $(A)-(D)$ respectively, with corresponding broadside patterns for the same array in $(D)-(G)$. Close to the array, the endfire beamwidth increases with a reducing range, but even at a range of just $10 \mathrm{~cm}$, directivity is maintained, although the beamwidth increases to $45^{\circ}$. For a comparison, the broadside array pattern, although initially much narrower is beginning to spread at a $1 \mathrm{~m}$ range, is as broad as the endfire pattern at $30 \mathrm{~cm}$ and has lost directivity altogether at $10 \mathrm{~cm}$. These near-field effects begin to occur at ranges less than about $l^{2} / 4 \lambda$ in broadside arrays, where $l$ is the array length and $\lambda$ is the wavelength.

In evolutionary terms, it is advantageous for a dolphin to maintain sonar contact with its target until it is close enough to grab the prey and swallow it. Similarly, there are many other sonar scenarios where operation down to very a short range is desirable. An obvious case is a diver hunting mines with a hand-held sonar.

\subsection{Monopulse localization}

Monopulse is a concept referring to precision direction finding with a pulsed source of radiation (Rhodes 1959). The direction of the pulsed source, whether it is a scattering target or an active beacon, is determined by simultaneously comparing the signals detected via two or more receiver beams. The main reason for its development was that signals that are fluctuating, for whatever reason, might lead to significant errors in receiving systems that require many pulses to be processed to extract directional information. If the angular measurement is based on one pulse rather than many, however, pulse-to-pulse amplitude fluctuations of the signal have little or no effect on angular accuracy.

Although strictly a receiving concept, monopulse has been applied primarily in the field of an active radar, and to a lesser extent, an active sonar. A basic explanation of the operating principles, along with typical system descriptions, will be found in any competent radar textbook (e.g. Kraus and Marhefka 2002).

Monopulse systems as described by Rhodes (1959) may operate by an amplitude comparison of squinted beams, as shown schematically in figure $8(A)$, or by a phase comparison between two displaced receiving elements, as in $8(B)$ (squint is a radar term referring to two beam patterns which diverge by a small angle - the squint angle). This comparison may be either additive or multiplicative. In the so-called sum-anddifference implementation, the difference between the left and right beam outputs, $P_{\mathrm{L}}$ and $P_{\mathrm{R}}$ (the 'difference beam'), is also found to be proportional to $\theta$ over a limited range. To make the output independent of variations in signal strength, the result is normalized by dividing by the sum of $P_{\mathrm{L}}$ and $P_{\mathrm{R}}$ (the 'sum beam') or some other representation of the received level.

The normal phase comparison implementation is essentially an interferometer and, again, the output is approximately proportional to $\theta$ over a limited range.

Variations of these methods are used in normal mammalian hearing (Moore 1997), and in biological contexts phase or time comparison is usually referred to as inter-aural time difference (ITD) and amplitude comparison as inter-aural intensity difference (IID), respectively.

\subsection{Monopulse and endfire combined}

The monopulse difference pattern obtained from two endfire beams is shown in figure 9. Here, the endfire arrays are made up of 22 elements with $1 \mathrm{~cm}$ spacing (as for figure 6); the frequency is $120 \mathrm{kHz}$ and the squint angle is $15^{\circ}$. The beam patterns are plotted on Cartesian axes in $(A)$ and the difference pattern is plotted as the solid line in $(B)$.

The dashed line in $(B)$ is a linear fit to the curve over the range $\pm 7^{\circ}$, and it is clearly seen that the difference pattern is close to linear over this range, referred to as the linear field of view. In fact, the deviation from linear is less than $\pm 0.1^{\circ}$ over $\pm 8^{\circ}$

There is a turning point in the pattern at about $\pm 10^{\circ}$, and beyond this point, it is no longer possible to determine the direction of arrival. This defines the unambiguous field of view. However, both the linear range and the unambiguous field of view are wider than the dolphin's transmitting beamwidth of $10^{\circ}$ (Au 1993), so normally no echoes are received outside the linear range. Thus, the system is in principle capable of unambiguously localizing a target to an accuracy of a tenth of a degree. In practice, noise and asymmetry between the two rows of teeth limit performance, and bottlenose dolphins are found to have 

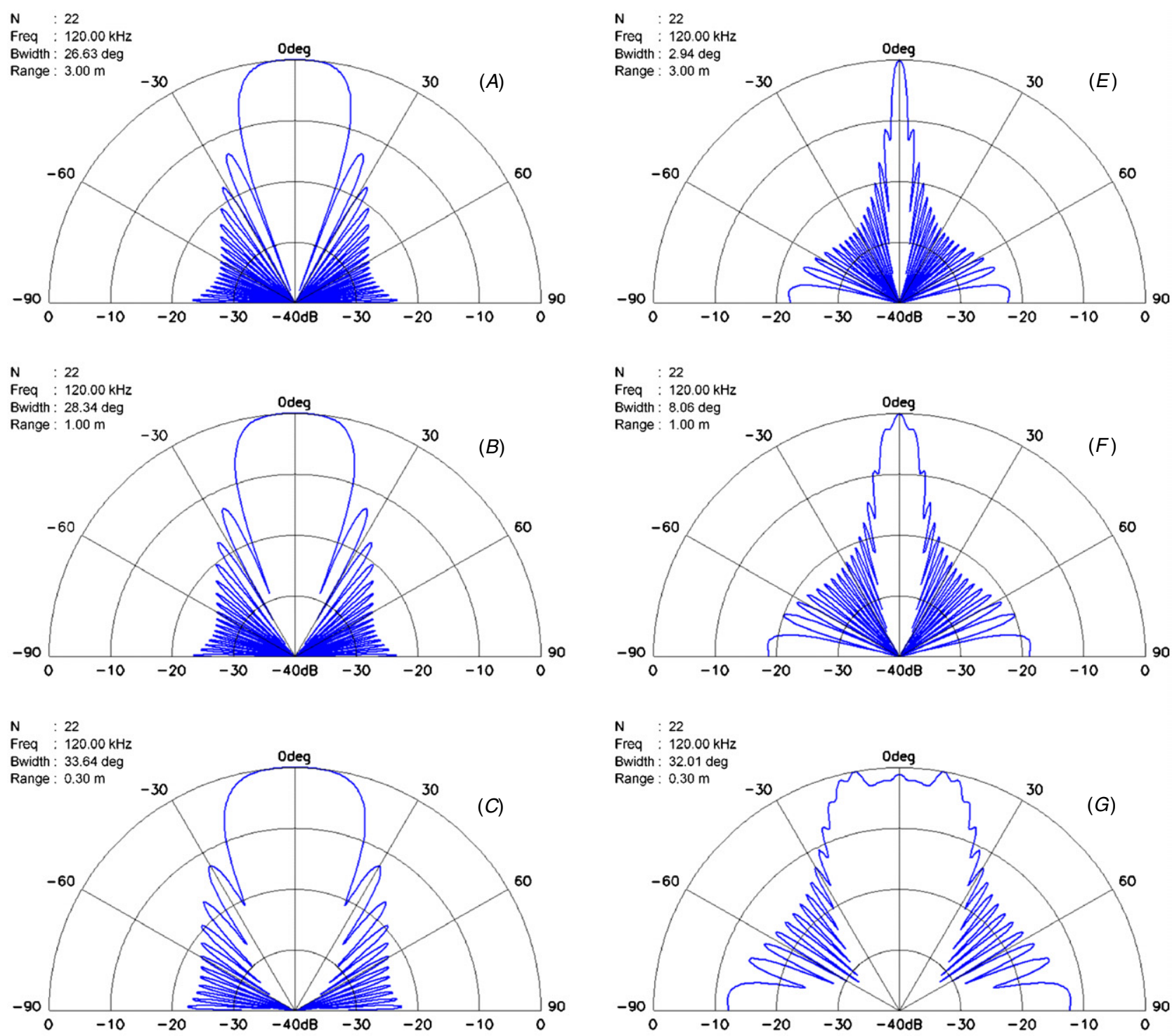

$\begin{array}{lll}\mathbf{N} & : 22 \\ \text { Freq }: & 120.00 \mathrm{kHz}\end{array}$
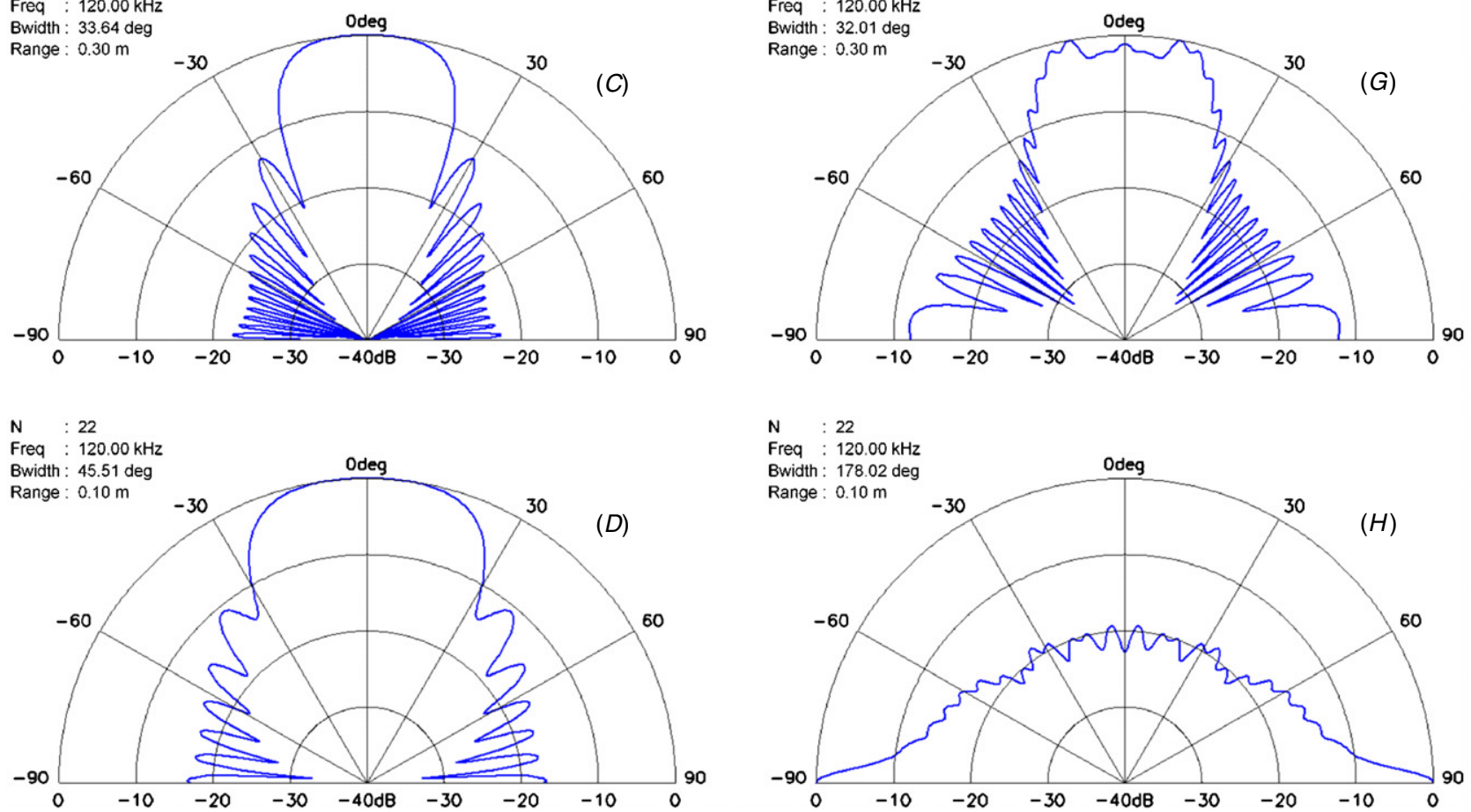

Figure 7. Near-field beam patterns at $120 \mathrm{kHz}$ for endfire array $(A)-(D)$, and the same array in the broadside mode in $(E)-(H)$. The range reduces from $3 \mathrm{~m}$ at the top to $10 \mathrm{~cm}$ at the bottom.

an accuracy and resolution of about $\pm 1^{\circ}$ when actively echolocating (Branstetter et al 2003).

There is, however, one limitation in this approach that may possibly lead to problems with broadband signals: the beamwidth of the endfire directivity pattern varies with frequency. The effect this has on the monopulse response is shown in figure 10, where difference patterns for the 22 element arrays are plotted for frequencies from $50 \mathrm{kHz}$ to $120 \mathrm{kHz}$. The slope of the difference pattern varies, and the linearity is degraded with reducing frequency.

This feature may not be significant in a homing system that changes direction to keep the target in the boresight direction, 

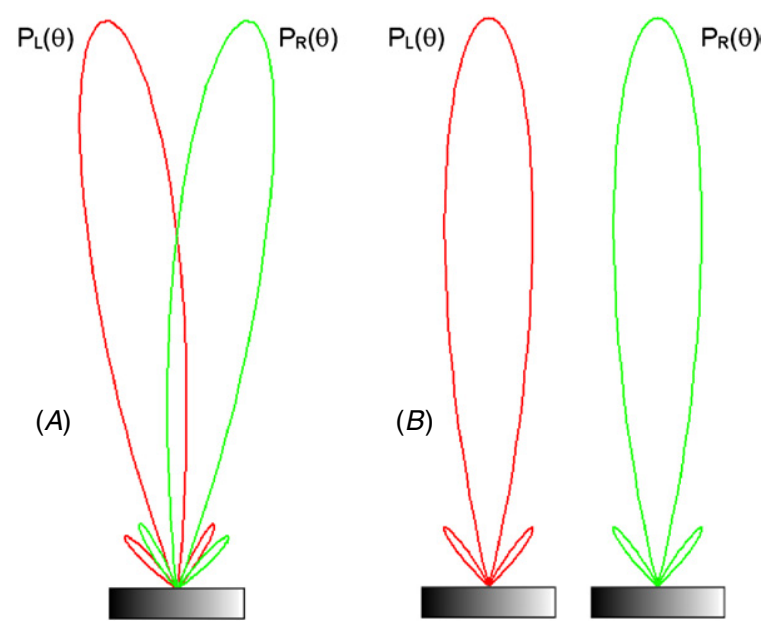

Figure 8. The two main monopulse configurations. Co-located squinted beams in $(A)$ for an amplitude comparison and displaced receivers in $(B)$ for a phase or time difference comparison.

thus maintaining a monopulse output close to zero. However, if the aim is to determine the direction of a target relative to the boresight over a wide frequency band, this system is not ideal. An alternative solution is suggested by certain species of river dolphin.

\section{River dolphins}

As already noted, the teeth of many dolphin species are linearly arranged with a constant inter-tooth dimension that appears related to the highest frequency used by their sonar. It is worth comparing this with the different structure developed by some river dolphins. There are currently four extant families that make up the river dolphins: the Platanistidae (Ganges and Indus River Dolphin), the Iniidae (Amazon River Dolphin or Boto), the Lipotidae (Chinese River Dolphin or Baiji) and the Pontoporiidae (La Plata Dolphin or Franciscana), but it is almost certain that within a decade the Lipotidae will have perished.

These animals all live in wide, shallow, murky rivers; many of them are blind and, generally, they possess a long and

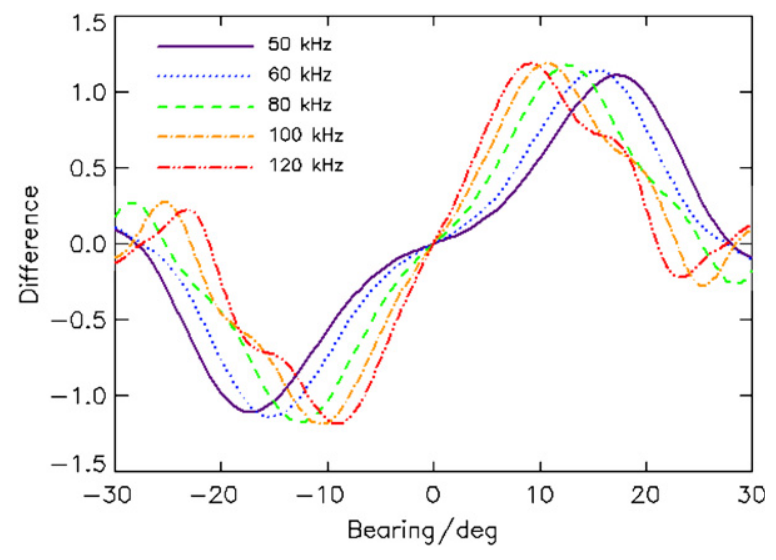

Figure 10. Difference patterns for the pair of 22 element array computed at frequencies from $50 \mathrm{kHz}$ to $120 \mathrm{kHz}$, showing the variation with frequency.

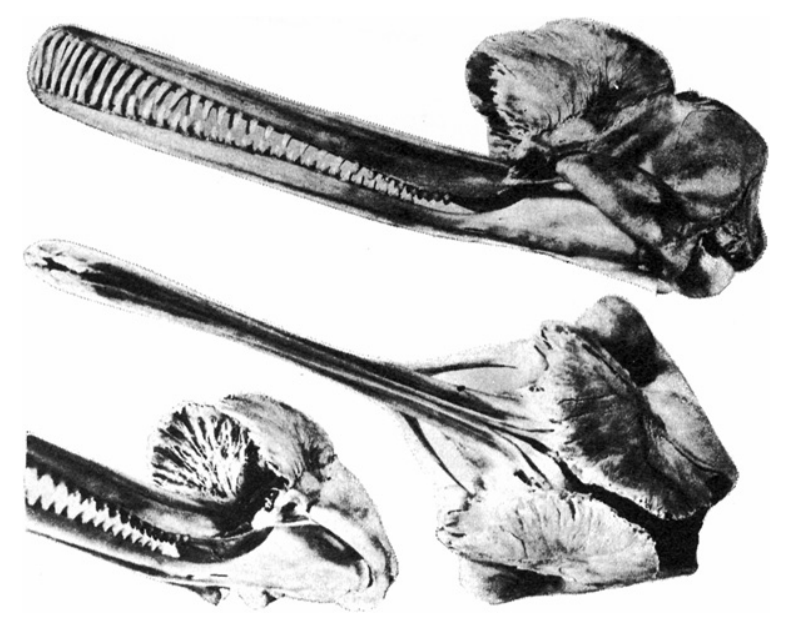

Figure 11. Photographs of Platanista skull (from Herald et al 1969) showing variation in tooth size and spacing along the jaw.

slender rostrum and jaw that support an unusual array of teeth. These teeth are longer and more closely spaced towards the tip. A precise description of the tooth arrangement is not available, but from images, such as figure 11 (from Herald et al 1969),
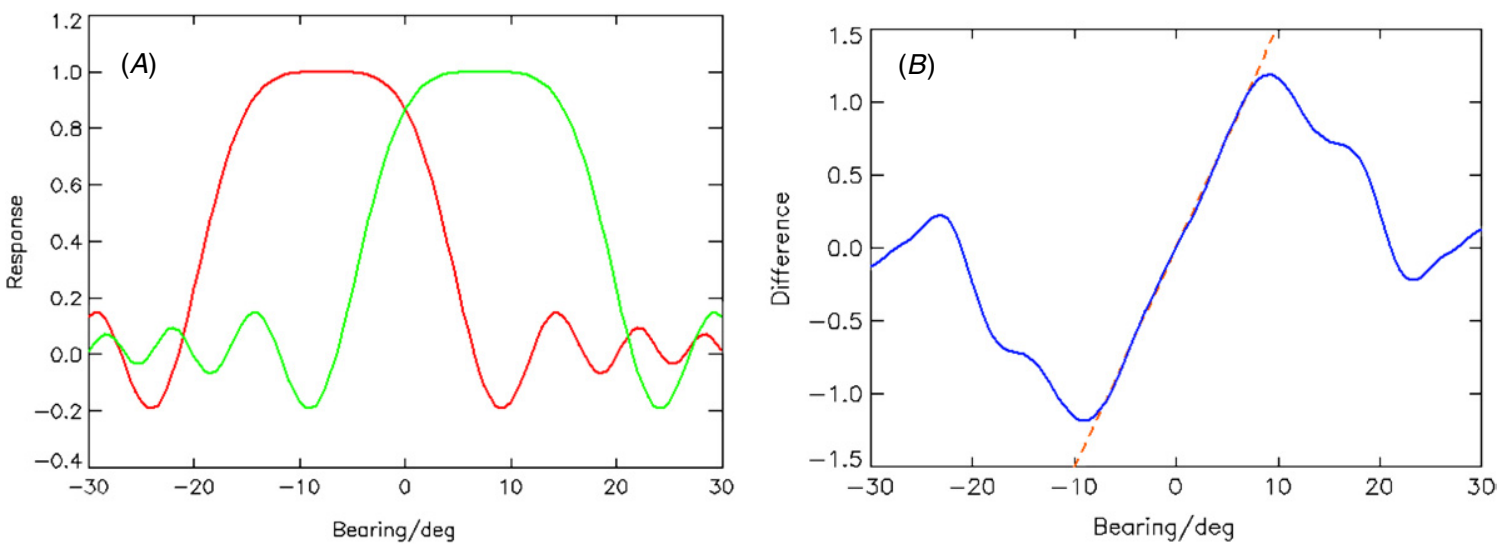

Figure 9. Endfire beams computed for a pair of 22 element array with $1 \mathrm{~cm}$ spacing, a squint angle of $15^{\circ}$ and a frequency of $120 \mathrm{kHz}(A)$, and the difference pattern, plotted as the solid line, with a linear fit over the range $\pm 7^{\circ}$ plotted as the dashed line $(B)$. 


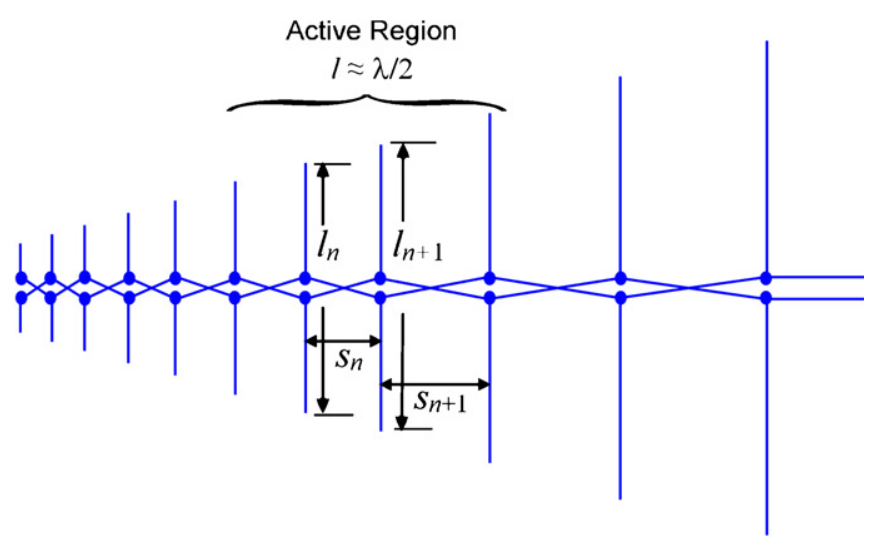

Figure 12. Sketch of log-periodic antenna.

and some inexact field measurements (Adloff and Schnapp 1986), there is a suggestion of a log-periodic distribution.

\subsection{Log-periodic arrays}

An RF log-periodic antenna array consists of a line array of dipoles which increase in length, $l$, and spacing, $s$, by a constant scaling factor, $k$, following a rule such as $l_{n+1} / l_{n}=$ $s_{n+1} / s_{n}=k$. This is shown schematically in figure 12 . Because dipoles are resonant at a frequency depending upon their length, this produces an active region comprising dipoles nearest resonance, which moves along the array with changing frequency.

With appropriate spacing, a log-periodic endfire array can be formed that has a constant active length in wavelengths, and hence a constant beamwidth, over a wide frequency band. Furthermore, the bell-shaped resonance curve effectively weights the array to produce a low sidelobe beam pattern.

Design charts and formulae for log-periodic arrays will be found in many radar textbooks (e.g. Kraus and Marhefka 2002), and these are equally applicable to acoustic implementations. The only significant difference is that hydrophones are not normally operated in a resonant mode, so the resonance response must be represented by a band-pass filter.

It might be informative to evaluate a log-periodic array based on a river dolphin tooth geometry and frequency range.
Table 1. Design parameters for an example log-periodic array.

\begin{tabular}{ll}
\hline No of elements & 78 \\
Minimum spacing/m & 0.003125 \\
Maximum resonant frequency $/ \mathrm{kHz}$ & 240 \\
Resonance $Q$ & 0.35 \\
Expansion factor $k$ & 1.03 \\
Overall length/m & 0.91 \\
\hline
\end{tabular}

However, for a comparison with the endfire array results shown in figures 6, 7, 9 and 10, a design configuration has been chosen to give an array with the same bandwidth and the same beamwidth as the bottlenose dolphin's at a frequency of $120 \mathrm{kHz}$, the top of its frequency range. This leads to the design parameters listed in table 1.

This design is given as an example, and no attempt at optimization has been made. It is likely that the large number of elements and an overall length of nearly a metre would not be suitable for some of the small-scale applications suggested in the introduction, such as a diver hand-held sonar, especially when compared with the 22 element array with a length of $11 \mathrm{~cm}$ representing the bottlenose dolphin's teeth. However, it is also likely that there is much scope for improvement in the design.

To model the increasing dipole length of the log-periodic antenna, resonant frequency, fres, reduces along the array as spacing increases. This leads to the modified rule fres $_{n+1} \times$ fres $_{n}=s_{n+1} / s_{n}=k$. The frequency response of the $n$th element is modelled as a simple second-order resonance. Again, it is likely that there is scope for finding improved frequency response functions.

Beam patterns for this array are plotted in figure 13. This shows far-field patterns at frequencies of $50 \mathrm{kHz}$ and $120 \mathrm{kHz}$ on the left and right, respectively. It is immediately clear that these beam patterns change little over the frequency range and also that they are essentially sidelobe free. The beamwidth is almost constant at approximately $26^{\circ}$, the same as that of the 22 element endfire array at $120 \mathrm{kHz}$. This frequency invariant beam pattern should overcome the limitations of the endfire array discussed at the end of the previous section, provided all the advantageous features of the endfire array/monopulse combination are maintained.

The first of these advantages is the endfire array's nearfield performance, and figure 14 shows that the log-periodic
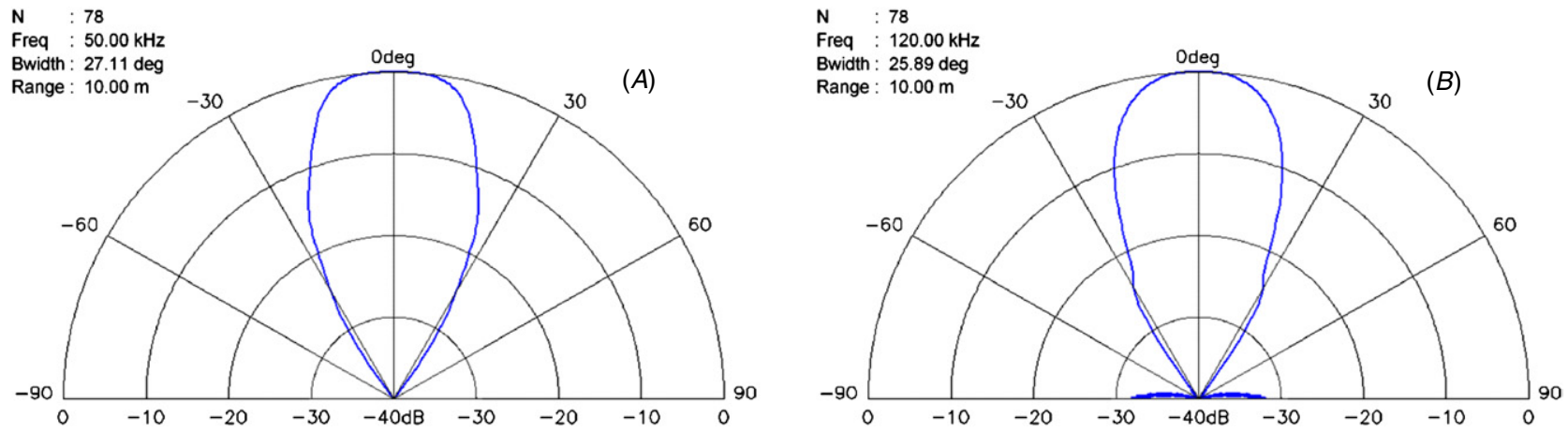

Figure 13. Log-periodic array far-field directivity patterns for $50 \mathrm{kHz}(A)$ and $120 \mathrm{kHz}(B)$. 

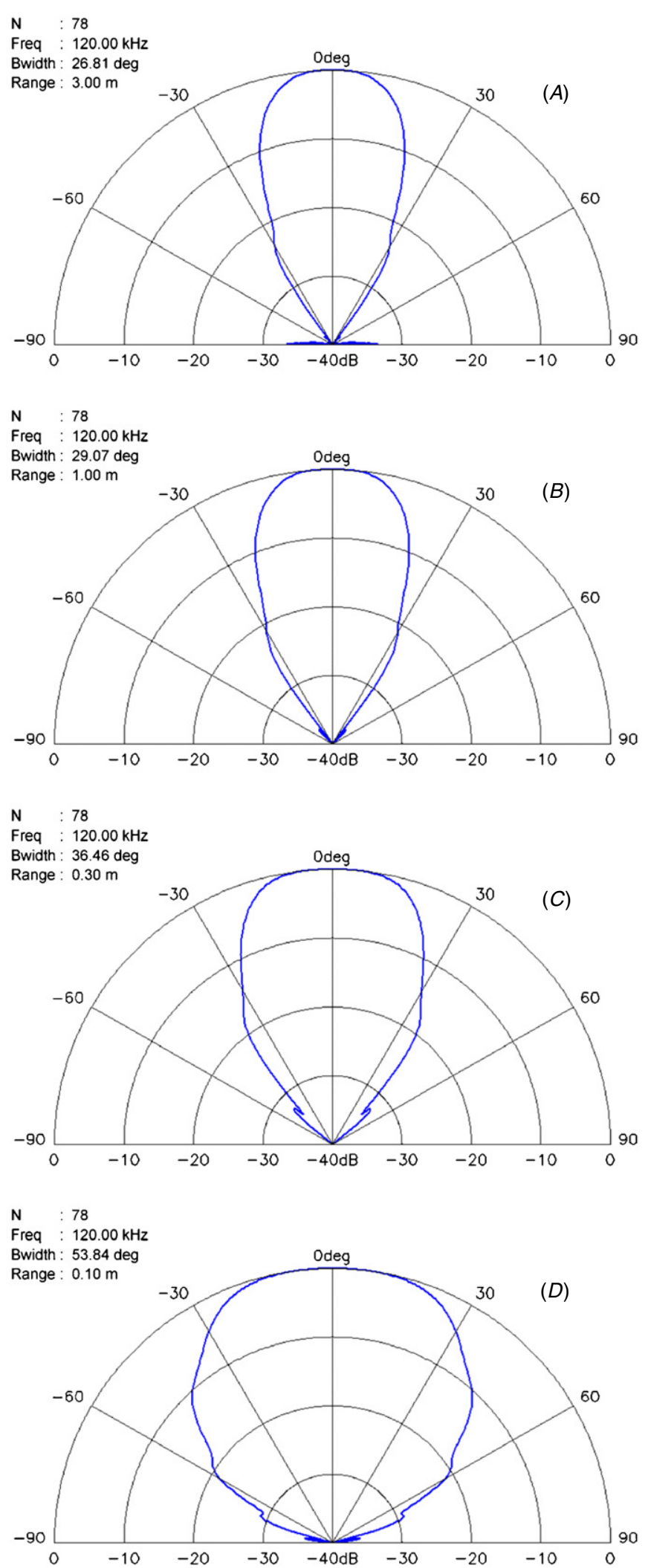

Figure 14. Near-field beam patterns at $120 \mathrm{kHz}$ for a log-periodic array. The range reduces from $3 \mathrm{~m}$ in $(A)$, through $1 \mathrm{~m}$ in $(B), 30 \mathrm{~cm}$ in $(C)$ to $10 \mathrm{~cm}$ in $(D)$.

array also maintains directivity at short ranges. Beam patterns at $120 \mathrm{kHz}$ are shown for ranges of $3 \mathrm{~m}, 1 \mathrm{~m}, 0.3 \mathrm{~m}$ and $0.1 \mathrm{~m}$.
As with the endfire case shown in figure 7, close to the array, the beamwidth increases with a reducing range, but even at a range of just $10 \mathrm{~cm}$ directivity is maintained.

The result of combining monopulse and log-periodic arrays is shown in figure 15. Difference patterns are plotted in $(A)$ for frequencies from $50 \mathrm{kHz}$ to $120 \mathrm{kHz}$ and the linear fit to the $120 \mathrm{kHz}$ curve over the range $\pm 12^{\circ}$ is also shown as a dashed line. Clearly, the difference patterns are close to linear over the entire frequency band, and the deviation from linearity is plotted for the same data in figure $15(B)$.

It can be seen that the deviation from linearity is less than $\pm 0.1^{\circ}$ over a field of view of $\pm 17^{\circ}$ from $50 \mathrm{kHz}$ to $120 \mathrm{kHz}$ although, as with the equispaced 22 element array, linearity is best at the highest frequencies. Nevertheless, it seems that the log-periodic array has entirely overcome the one important limitation of the endfire array.

\section{Discussion and conclusions}

This paper has described a new concept for a sonar receiver inspired by what little is known about dolphin echolocation receiving mechanisms. It should be stressed that no claims are made regarding the dolphin's receiving mechanisms and physiology; the principles described here would be equally useful if it were found that, in fact, the dolphin receiver was something else altogether.

The underlying ideas may be summarized as follows.

- Dolphins' teeth can be modelled as a pair of endfire arrays.

- Endfire array beam patterns have minimal near-field degradation.

- A pair of endfire arrays can be used in a monopulse mode for angular localization.

These three statements embody an idea for a sonar receiver that is put together from components that are familiar in sonar and radar technology. However, this particular combination has apparently not been tried in man-made systems as yet. Nevertheless, many of the toothed whales, after 35 million years of trial and error, may have settled on this configuration as the optimal sensor for detecting, classifying and localizing targets the size of a sardine over ranges from zero to $100 \mathrm{~m}$ or more in all sea states and all maritime environments from the open ocean through the surf zone to rivers and estuaries.

These characteristics should be considered in combination with other known features of dolphin echolocation. For example, the dolphin transmitter appears to produce sidelobefree beam patterns, minimizing the effect of off-axis reverberation. Dolphin signals give sub-cm range resolution. This means that, although the receiver has a wide beamwidth, allowing rapid area coverage, there is a low probability of more than one target appearing in a single range cell and confusing the monopulse process.

The performance of endfire arrays at a short range has been described. In poor visibility, it is clearly an advantage to maintain sonar contact when approaching a target so as to minimize the risk of losing the target through some lastminute evasive manoeuvre. Some conventional imaging sonars employ focusing to achieve short-range operation. 

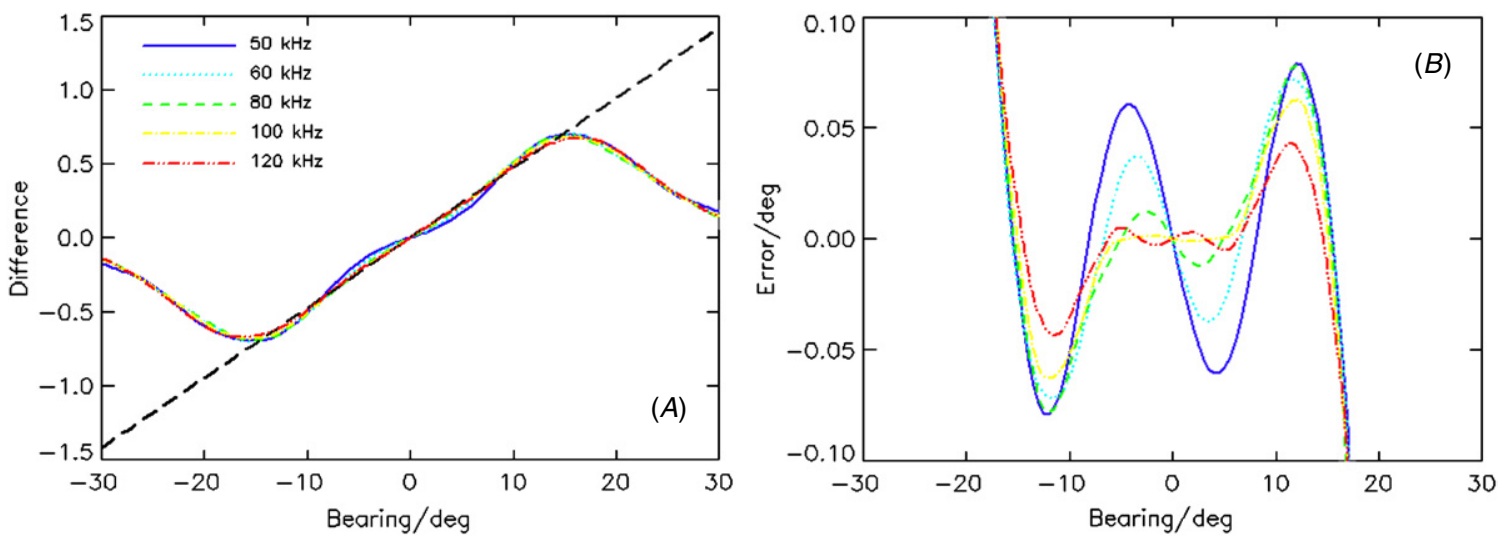

Figure 15. (A) Difference patterns for the pair of log-periodic arrays, with a squint angle $13^{\circ}$, and computed at frequencies from $50 \mathrm{kHz}$ to $120 \mathrm{kHz}$, showing negligible variation with frequency. $(A)$ Linear fit to the $120 \mathrm{kHz}$ curve over the range $\pm 12^{\circ}$ is plotted as a dashed line. $(B)$ Deviation from linearity for the same set of frequencies.

This, however, requires additional electronics, increasing the size, weight, power consumption and, of especial significance in military scenarios, the magnetic signature.

The one limitation of the endfire array, as with all conventional arrays, is that its beam pattern changes with frequency. This may not be desirable in a broadband system. However, this difficulty may be overcome using log-periodic arrays, which potentially have additional advantages of

- further reduced near-field variation,

- reduced sidelobes,

- negligible variation with frequency over a wide bandwidth.

Overall, the system considered here is potentially ideal for small, lightweight, high-resolution sonars capable of effective operation in shallow water environments, to be carried by divers or underwater vehicles. However, there is more to dolphin echolocation than just the receiver. It now remains to determine how the dolphins might combine such a receiver with their specific signal waveforms, transmitter characteristics and processing techniques to achieve such impressive detection, classification and localization performance with their sardine-sized targets.

\section{Acknowledgments}

Much of the work underlying this paper was carried out as student projects supervised by the author when he was in the Physics Department of the University of Bath over the period 2002-04. In particular, the contributions of Matthew Freeman and Robert Floyd were especially valuable.

\section{References}

Adloff D and Schnapp D 1986 Operation platanista private report (Copenhagen)

Au W W L 1993 The Sonar of Dolphins (New York: Springer-Verlag)

Au W W L 2004 Dolphin sonar detection and discrimination capabilities J. Acoust. Soc. Am. 1152614
Au W W L, Pawloski J L, Nachtigall P E, Blonz M and Gisner R C 1995 Echolocation signals and transmission beam pattern of a false killer whale (pseudorca crassidens) J. Acoust. Soc. Am. 98 51-9

Belcher E, Hanot W and Burch J 2002 Proc. 2002 Int. Symp. on Underwater Technology. pp 187-92

Berktay H O and Shooter J A 1973 Nearfield effects in end-fire line arrays J. Acoust. Soc. Am. $\mathbf{5 3} 550-6$

Branstetter B K, Mevissen S J, Herman L M, Pack A A and Roberts S P 2003 Horizontal angular discrimination by an echolocating bottlenose dolphin tursiops truncatus Bioacoustics 14 15-34

Brill R L, Moore P W B, Helweg D A and Dankiewicz L A 2000 Mapping acoustic sensitivity about the dolphin's head: a look at the peripheral hearing system J. Acoust. Soc. Am. 1072786

Capus C, Pailhas Y, Brown K, Lane D M, Moore P W and Houser D 2007 Bio-inspired wideband sonar signals based on observations of the bottlenose dolphin (Tursiops truncatus) J. Acoust. Soc. Am. 121 594-604

Commander K W, Christoff J T and Lopes J L 2002 Current development of high-resolution mcm minehunting sonars for small auvs J. Acoust. Soc. Am. $1112414-5$

Cook M L H, Wells R S and Mann D A 2004 Auditory brainstem response hearing measurements in free-ranging bottlenose dolphins (Tursiops truncatus) J. Acoust. Soc. Am. 1162504

Dible S A, Flint J A and Lepper P A 2006 8th European Conf. Underwater Acoustics (Carvoeiro, Portugal) ed S M Jesus and O C Rodriguez

Dobbins P F 2001 Modelling dolphin echolocation reception Proc. Inst. Acoust. 23 123-32

Goodson A D and Klinowska M A 1990 A proposed echolocation receptor for the Bottlenose Dolphin (Tursiops truncatus): modelling the receive directivity from tooth and lower jaw geometry Sensory Abilities of Cetaceans vol 196 ed J A Thomas and R A Kastelein (New York: Plenum) pp 255-67 (NATO ASI Series A)

Hallett J and Chapman S 2003 MCM-faster, better and integrated into task groups J. Def. Sci. 8 8-12

Herald E S, Brownwell R L, Frye F L, Evans W E and Scott A B 1969 Blind river dolphin: First side-swimming cetacean Science 166 1408-10

Johnson C S 1997 A window into the acoustics of whales and dolphins J. Acoust. Soc. Am. 1023101

Kastelein R A, Au W W L, Rippe H T and Schooneman N M 1999 Target detection by an echolocating harbor porpoise (phocoena phocoena) J. Acoust. Soc. Am. $1052493-8$

Kraus J and Marhefka R 2002 Antennas For All Applications (New York: McGraw-Hill) 
Mendes B, Fonseca P and Campos A 2004 Weight-length relationships for 46 fish species of the Portuguese west coast J. Appl. Ichthyol. 20 355-61

Moore B C J 1997 An Introduction to the Psychology of Hearing 4th edn (New York: Academic)

Nelson M 2005 The possible role of teeth in sperm whale (Physeter macrocephalus) echolocation MSc Thesis School of Biological Sciences, University of Wales, Bangor

Potter J R and Taylor E A 2001 On novel reception models for bottlenose dolphin echolocation Proc. Inst. Acoust. 23 103-12

Reeves R R, Smith B D, Crespo E A and di Sciara G N (Compilers) 2003 Dolphins, Whales and Porpoises: 2002-2010

Conservation Action Plan for the World's Cetaceans IUCN/SSC Cetacean Specialist Group, IUCN, Gland, Switzerland and Cambridge, UK

Rhodes D R 1959 Introduction to Monopulse (New York: McGraw-Hill)
Richards S and Leighton T 2003 High frequency sonar performance predictions for littoral operations - the effects of suspended sediments and microbubbles J. Def. Sci. 8 1-7

Ryabov V A 2004 Lower jaw-peripheric part of the dolphin echolocation hearing Proc. Marine Mammals of the Holarctic, 3rd Int. Conf. pp 483-9

Sank J R 1985 Microphones J. Audio Eng. Soc. 33 514-47

Sigurdson J E 1997a Analyzing the dynamics of dolphin biosonar behaviour during search and detection tasks Proc. Inst. Acoust. 19 123-32

Sigurdson J E 1997b Biosonar dynamics of the bottlenose dolphin in VSW search and detection tasks J. Acoust. Soc. Am. 1023123

Thomas J, Moss C and Vater M 2004 Echolocation in Bats and Dolphins (Chicago, IL: University of Chicago Press)

Ura T et al 2006 Estimates of bio-sonar characteristics of a free-ranging Ganges river dolphin J. Acoust. Soc. Am. 1203228 